\title{
ON THE DYNAMICS OF THE RIGID BODY WITH A FIXED POINT: PERIODIC ORBITS AND INTEGRABILITY
}

\author{
JUAN L.G. GUIRAO ${ }^{1}$, JAUME LLIBRE ${ }^{2}$ AND JUAN A. VERA ${ }^{3}$
}

\begin{abstract}
The aim of the present paper is to study the periodic orbits of a rigid body with a fixed point and quasi-spherical shape under the effect of a Newtonian force field given by different small potentials. For studying these periodic orbits we shall use averaging theory. Moreover, we provide information on the $\mathcal{C}^{1}$-integrability of these motions.
\end{abstract}

\section{Introduction And Statement of the main Results}

The dynamics of rigid bodies, in its different formulations Eulerian, Lagrangian and Hamiltonian, has been extensively studied in the classic literature, see for instance [9] for a classic treatment of these topics or [1] for a more moder approach.

The main objectives in the study of the motion of a rigid body with a fixed point are:

(1) To state the equilibria and their stabilities in rigid bodies with a fixed point.

(2) To state the periodic solutions, bifurcations and chaos of its motion.

(3) To analyze the integrability and to state the first integrals for the problem.

Our main aim in this work is to study the periodic orbits of a rigid body with a fixed point under a Newtonian gravitational field using averaging theory. As a corollary we also obtain information about $\mathcal{C}^{1}$ non-integrability.

It is well known, see for instance [3, page 57], that the motion of a rigid body with a fixed point is described by the Hamiltonian equations associated to the Hamiltonian

$$
\mathcal{H}=\frac{\left(G^{2}-L^{2}\right)}{2}\left(\frac{\sin ^{2} l}{A}+\frac{\cos ^{2} l}{B}\right)+\frac{L^{2}}{C}+U\left(k_{1}, k_{2}, k_{3}\right)
$$

with

$$
k_{1}=\left(\frac{H}{G} \sqrt{1-\left(\frac{L}{G}\right)^{2}}+\frac{L}{G} \sqrt{1-\left(\frac{H}{G}\right)^{2}} \cos g\right) \sin l+
$$

Key words and phrases. Rigid body with a fixed point, periodic orbits, integrability, averaging theory.

2010 Mathematics Subject Classification. Primary: 70E17, 70E20, 70E40. Secondary: $37 \mathrm{C} 27$. 


$$
\begin{gathered}
\sqrt{1-\left(\frac{H}{G}\right)^{2}} \sin g \cos l \\
k_{2}=\left(\frac{H}{G} \sqrt{1-\left(\frac{L}{G}\right)^{2}}+\frac{L}{G} \sqrt{1-\left(\frac{H}{G}\right)^{2}} \cos g\right) \cos l- \\
\sqrt{1-\left(\frac{H}{G}\right)^{2}} \sin g \sin l \\
k_{3}=\left(\frac{H}{G}\right)\left(\frac{L}{G}\right)-\sqrt{1-\left(\frac{L}{G}\right)^{2}} \sqrt{1-\left(\frac{H}{G}\right)^{2}} \cos g
\end{gathered}
$$

This is a Hamiltonian in the Andoyer-Deprit canonical variables $(L, G, l, g)$ of two degree of freedom with the positive parameters $A, B, C$ and $H$.

As it is usual we introduced the parameters

$$
\alpha=\frac{\frac{1}{A}+\frac{1}{B}-\frac{2}{C}}{\frac{2}{C}}, \quad \beta=\frac{\frac{1}{A}-\frac{1}{B}}{\frac{1}{A}+\frac{1}{B}-\frac{2}{C}}
$$

The parameter $\beta$ is known as the triaxial coefficient. Note that $\alpha$ can take any positive value depending on the physical characteristics of the rigid body. But the triaxial coefficient $\beta$ is bounded between zero (the oblate spheroid $A=B$ ) and one (the prolate spheroid $B=C$ ), although it is undefined in the limit case of a sphere, taking any value between zero and one depending on the direction in which we approach the limit. See for more details [7].

In this work we assume that $0<\alpha=\varepsilon^{k} \ll 1$, i.e. Then the Hamiltonian (1) is expressed by

where

$$
\mathcal{H}=\frac{G^{2}}{2 C}+\varepsilon^{k} \mathcal{P}_{1}+U\left(k_{1}, k_{2}, k_{3}\right)
$$

$$
\mathcal{P}_{1}=\frac{1}{2 C}\left(G^{2}-L^{2}\right)(1-\beta \cos 2 l) .
$$

Moreover we shall consider the following three cases:

Case 1: $U\left(k_{1}, k_{2}, k_{3}\right)=\varepsilon V\left(k_{1}, k_{2}, k_{3}\right)$ and $k=2$, i.e.

$$
\mathcal{H}=\frac{G^{2}}{2 C}+\varepsilon \mathcal{P}_{2}+\varepsilon^{2} \mathcal{P}_{1},
$$

where $\mathcal{P}_{2}=V\left(k_{1}, k_{2}, k_{3}\right)$.

Case 2: $U\left(k_{1}, k_{2}, k_{3}\right)=\varepsilon V\left(k_{1}, k_{2}, k_{3}\right)$ and $k=1$, i.e.

$$
\mathcal{H}=\frac{G^{2}}{2 C}+\varepsilon\left(\mathcal{P}_{1}+\mathcal{P}_{2}\right) .
$$

Case 3: $U\left(k_{1}, k_{2}, k_{3}\right)=\varepsilon^{2} V\left(k_{1}, k_{2}, k_{3}\right)$ and $k=1$, i.e.

$$
\mathcal{H}=\frac{G^{2}}{2 C}+\varepsilon \mathcal{P}_{2}+\varepsilon^{2} \mathcal{P}_{1} \text {. }
$$


We note that $\mathcal{P}_{1}$ measures the difference of the shape of the rigid body between a sphere and a tri-axial ellipsoid, and $\mathcal{P}_{2}$ measures the external forces acting on the rigid body. We shall assume that the perturbing functions $\mathcal{P}_{i}$ are smooth in the variables $(L, l ; G, g)$.

Our main results are the following three theorems each one studying the periodic orbits of the previous three cases.

Theorem 1. We consider the motion of the rigid body with a fixed point under the action of the Hamiltonian (2). On the energy level $\mathcal{H}=h>0$ if $\varepsilon \neq 0$ is sufficiently small, then for every zero $\left(L_{0}, l_{0}\right)$ of the system

$$
\begin{aligned}
& f_{1}^{1}(L, l)=-\frac{1}{2 \pi} \sqrt{\frac{C}{2 h}} \int_{0}^{2 \pi} \frac{\partial \mathcal{P}_{2}}{\partial l} d g=0, \\
& f_{1}^{2}(L, l)=\frac{1}{2 \pi} \sqrt{\frac{C}{2 h}} \int_{0}^{2 \pi} \frac{\partial \mathcal{P}_{2}}{\partial L} d g=0,
\end{aligned}
$$

satisfying that

$$
\operatorname{det}\left(\left.\frac{\partial\left(f_{1}^{1}, f_{1}^{2}\right)}{\partial(L, l)}\right|_{(L, l)=\left(L_{0}, l_{0}\right)}\right) \neq 0,
$$

there exists a $2 \pi$-periodic solution $(L(g, \varepsilon), l(g, \varepsilon), G(g, \varepsilon))$ in the variable $g$ of the rigid body such that $(L(g, 0), l(g, 0), G(g, 0))=\left(L_{0}, l_{0}, \sqrt{2 C h}\right)$ when $\varepsilon \rightarrow 0$.

Let $R=\left(a^{2}-b^{2}\right)^{2}\left(a^{2}+b^{2}\right)+\left(a^{4}-6 a^{2} b^{2}+b^{4}\right) c^{2}$. An application of Theorem 1 is the following.

Corollary 2. A spherical rigid body with Hamiltonian (2), weak potential $\mathcal{P}_{2}=$ $a k_{1}+b k_{2}+c k_{3}$ with $a, b$ and $c$ positive and $\varepsilon \neq 0$ sufficiently small has in every positive energy level at least four linear stable periodic orbits if $R>0$, two linear stable periodic orbits if $R=0$, and two linear stable periodic orbits and two unstable ones if $R<0$.

Note that Corollary 2 describes the motion of a non-homogeneous sphere with center of mass at the point $(a, b, c)$ under a weak gravitational Newtonian potential.

Theorem 3. We consider the motion of the rigid body with a fixed point under the action of the Hamiltonian (3). On the energy level $\mathcal{H}=h>0$ if $\varepsilon \neq 0$ is sufficiently small, then for every zero $\left(L_{0}, l_{0}\right)$ of the system

$$
\begin{aligned}
& f_{1}^{1}(L, l)=-\frac{1}{2 \pi} \sqrt{\frac{C}{2 h}} \int_{0}^{2 \pi} \frac{\partial\left(\mathcal{P}_{1}+\mathcal{P}_{2}\right)}{\partial l} d g=0, \\
& f_{1}^{2}(L, l)=\frac{1}{2 \pi} \sqrt{\frac{C}{2 h}} \int_{0}^{2 \pi} \frac{\partial\left(\mathcal{P}_{1}+\mathcal{P}_{2}\right)}{\partial L} d g=0,
\end{aligned}
$$

satisfying (5), there exists a $2 \pi$-periodic solution $(L(g, \varepsilon), l(g, \varepsilon), G(g, \varepsilon))$ in the variable $g$ of the rigid body such that $(L(g, 0), l(g, 0), G(g, 0))=\left(L_{0}, l_{0}, \sqrt{2 C h}\right)$ when $\varepsilon \rightarrow 0$. 
An application of Theorem 3 is the following.

Corollary 4. A quasi-spherical rigid body with Hamiltonian (3), weak potential $\mathcal{P}_{2}=c k_{3}$ with $c>0$ and $\varepsilon \neq 0$ sufficiently small can have at least eight periodic orbits in every positive energy level.

We remark that Corollary 4 describes the motion of a non-homogeneous quasi-spherical rigid body with center of mass at the point $(0,0, c)$ under a weak gravitational Newtonian potential. The linear stability of the periodic orbits described in Corollary 4 can be easily studied using Theorem 10 but since there are many possibilities we do not present an explicit description here.

Theorem 5. We consider the motion of the rigid body with a fixed point under the action of the Hamiltonian (4). On the energy level $\mathcal{H}=h>0$ if $\varepsilon \neq 0$ is sufficiently small and

$$
\int_{0}^{2 \pi} \frac{\partial \mathcal{P}_{1}}{\partial l}=\int_{0}^{2 \pi} \frac{\partial \mathcal{P}_{1}}{\partial L}=0
$$

then for every zero $\left(L_{0}, l_{0}\right)$ of the system

$$
\begin{aligned}
& f_{2}^{1}(L, l)=\frac{1}{2 \pi} \int_{0}^{2 \pi} F_{2}^{1}(L, l, g) d g=0, \\
& f_{2}^{2}(L, l)=\frac{1}{2 \pi} \int_{0}^{2 \pi} F_{2}^{2}(L, l, g) d g=0,
\end{aligned}
$$

where

$$
\begin{gathered}
F_{2}^{1}=\frac{1}{4 h^{2}}\left(2 h C\left(-\frac{\partial^{2} \mathcal{P}_{1}}{\partial l^{2}}\left(\int_{0}^{g} \frac{\partial \mathcal{P}_{1}}{\partial L} d g\right)+\frac{\partial^{2} \mathcal{P}_{1}}{\partial L \partial l}\left(\int_{0}^{g} \frac{\partial \mathcal{P}_{1}}{\partial l} d g\right)\right)+\sqrt{\frac{C}{(2 h)^{3}}} \mathcal{P}_{1}\right. \\
\left(-\frac{\partial \mathcal{P}_{1}}{\partial l}+\sqrt{2 C h} \frac{\partial^{2} \mathcal{P}_{1}}{\partial l \partial G}\right)+\frac{C}{2 h} \frac{\partial \mathcal{P}_{1}}{\partial G} \frac{\partial \mathcal{P}_{1}}{\partial l}-\sqrt{\left.\frac{C}{2 h} \frac{\partial \mathcal{P}_{2}}{\partial l}\right)}, \\
F_{2}^{2}=\frac{1}{4 h^{2}}\left(2 h C\left(-\frac{\partial^{2} \mathcal{P}_{1}}{\partial L^{2}}\left(\int_{0}^{g} \frac{\partial \mathcal{P}_{1}}{\partial l} d g\right)+\frac{\partial^{2} \mathcal{P}_{1}}{\partial L \partial l}\left(\int_{0}^{g} \frac{\partial \mathcal{P}_{1}}{\partial L} d g\right)\right)+\sqrt{\frac{C}{(2 h)^{3}}} \mathcal{P}_{1}\right. \\
\left.\left(\frac{\partial \mathcal{P}_{1}}{\partial L}-\sqrt{2 C h} \frac{\partial^{2} \mathcal{P}_{1}}{\partial L \partial G}\right)-\frac{C}{2 h} \frac{\partial \mathcal{P}_{1}}{\partial G} \frac{\partial \mathcal{P}_{1}}{\partial L}+\sqrt{\frac{C}{2 h}} \frac{\partial \mathcal{P}_{2}}{\partial L}\right),
\end{gathered}
$$

satisfying (5), there exists a $2 \pi$-periodic solution $(L(g, \varepsilon), l(g, \varepsilon), G(g, \varepsilon))$ in the variable $g$ of the rigid body such that $(L(g, 0), l(g, 0), G(g, 0))=\left(L_{0}, l_{0}, \sqrt{2 C h}\right)$ when $\varepsilon \rightarrow 0$.

An application of Theorem 5 is the following.

Corollary 6. A quasi-spherical rigid body with Hamiltonian (4), weak potential $\mathcal{P}_{2}=c k_{3}^{2}$ with $c>0$, energy level $\mathcal{H}=h=3 H^{2} /(2 C)$ and $\varepsilon \neq 0$ sufficiently small can have at least fourteen periodic solutions. 
In the proofs of Theorems 1 and 3 we shall use the averaging theory of first order, and in the proof of Theorem 5 we shall use the averaging theory of second order. We note that the averaging method allows to find analytically periodic orbits of the rigid body with a fixed point at any positive energy level, reducing its study to find zeros of a convenient system of two equations with two unknowns.

We shall use the periodic orbits found in the previous theorems for obtaining information about the $\mathcal{C}^{1}$ non-integrability in the sense of Liouville-Arnold of a rigid body with a fixed point.

Theorem 7. For a rigid body with a fixed point whose motion is given by the Hamiltonian (2) (respectively (3) or (4)) we have:

(1) Either the Hamiltonian system is Liouville-Arnold integrable and the gradients of two independent first integrals are linearly dependent on some points of all the periodic orbits found in Theorem 1 (respectively 3 or 5);

(2) or it is not Liouville-Arnold integrable with any second first integral of class $\mathcal{C}^{1}$.

Similar studies on the periodic obits and on the integrability of the Hamiltonian systems for the Henon-Heiles Hamiltonian and the Yang-Mills Hamiltonian have been done in [5] and [6] respectively.

The rest of the paper is dedicated to the proofs of theorems and corollaries stated in this introduction.

\section{Proof of Theorems 1,3 And 5}

Proof of Theorem 1. The Hamiltonian differential equations associated to the Hamiltonian 2 are

$$
\begin{aligned}
\frac{d L}{d t} & =-\varepsilon \frac{\partial \mathcal{P}_{2}}{\partial l}+O\left(\varepsilon^{2}\right), & \frac{d G}{d t} & =-\varepsilon \frac{\partial \mathcal{P}_{2}}{\partial g}+O\left(\varepsilon^{2}\right), \\
\frac{d l}{d t} & =\varepsilon \frac{\partial \mathcal{P}_{2}}{\partial L}+O\left(\varepsilon^{2}\right), & \frac{d g}{d t} & =\frac{G}{C}+\varepsilon \frac{\partial \mathcal{P}_{2}}{\partial G}+O\left(\varepsilon^{2}\right) .
\end{aligned}
$$

Taking $g$ as the new independent variable the previous differential systems becomes

$$
\frac{d L}{d g}=-\varepsilon \frac{C}{G} \frac{\partial \mathcal{P}_{2}}{\partial l}+O\left(\varepsilon^{2}\right), \frac{d G}{d g}=-\varepsilon \frac{C}{G} \frac{\partial \mathcal{P}_{2}}{\partial g}+O\left(\varepsilon^{2}\right), \frac{d l}{d g}=\varepsilon \frac{C}{G} \frac{\partial \mathcal{P}_{2}}{\partial L}+O\left(\varepsilon^{2}\right) .
$$

Since we want to study the periodic orbits on the energy level $\mathcal{H}=h$ we isolated $G$ from the energy, obtaining $G=\sqrt{2 h C}+O(\varepsilon)$ because $G$ is the modulus of the angular momentum. Therefore, the equations of motion on this energy level are

$$
\frac{d L}{d g}=-\varepsilon \sqrt{\frac{C}{2 h}} \frac{\partial \mathcal{P}_{2}}{\partial l}+O\left(\varepsilon^{2}\right), \frac{d l}{d g}=\varepsilon \sqrt{\frac{C}{2 h}} \frac{\partial \mathcal{P}_{2}}{\partial L}+O\left(\varepsilon^{2}\right) .
$$


These differential equations are in the normal form for applying the first order averaging theory, see Theorem 10. From this theorem the proof is over.

Proof of Corollary 2. For proving Corollary 2 from Theorem 1 we need to compute $\left(f_{1}^{1}, f_{1}^{2}\right)$. Indeed we have

$$
\begin{gathered}
f_{1}=\left(f_{1}^{1}, f_{1}^{2}\right)=\left(\frac{H \sqrt{4-\frac{2 L^{2}}{C h}}(b \sin l-a \cos l)}{4 h},\right. \\
\left.\frac{c \sqrt{C h} \sqrt{2-\frac{L^{2}}{C h}}-H L \sqrt{4-\frac{2 L^{2}}{C h}}(b \cos l+a \sin l)}{2 C h^{2} \sqrt{4-\frac{2 L^{2}}{C h}}}\right) .
\end{gathered}
$$

Solving the system $\left(f_{1}^{1}, f_{1}^{2}\right)=(0,0)$ we obtain the following four solutions

$$
\left(L= \pm \frac{c \sqrt{2 C h}}{\sqrt{a^{2}+b^{2}+c^{2}}}, l= \pm \arccos \left(\frac{b}{\sqrt{a^{2}+b^{2}}}\right)\right) .
$$

On the other hand, the determinant (5) for the four solutions is equal to

or

$$
\frac{\left(a^{2}+b^{2}+c^{2}\right) H^{2}}{8 c h^{3}}
$$

$$
\frac{\left(\left(a^{2}-b^{2}\right)^{2}\left(a^{2}+b^{2}\right)+\left(a^{4}-6 a^{2} b^{2}+b^{4}\right) c^{2}\right) H^{2}}{8\left(a^{2}+b^{2}\right)^{2} C h^{3}} .
$$

Analyzing the eigenvalues of the matrix which appear in (5) the corollary follows.

Proof of Theorem 3. The Hamiltonian differential equations associated to the Hamiltonian 3 are

$$
\begin{array}{ll}
\frac{d L}{d t}=-\varepsilon \frac{\partial\left(\mathcal{P}_{1}+\mathcal{P}_{2}\right)}{\partial l}+O\left(\varepsilon^{2}\right), & \frac{d G}{d t}=-\varepsilon \frac{\partial\left(\mathcal{P}_{1}+\mathcal{P}_{2}\right)}{\partial g}+O\left(\varepsilon^{2}\right), \\
\frac{d l}{d t}=\varepsilon \frac{\partial\left(\mathcal{P}_{1}+\mathcal{P}_{2}\right)}{\partial L}+O\left(\varepsilon^{2}\right), & \frac{d g}{d t}=\frac{G}{C}+\varepsilon \frac{\partial\left(\mathcal{P}_{1}+\mathcal{P}_{2}\right)}{\partial G}+O\left(\varepsilon^{2}\right) .
\end{array}
$$

Taking $g$ as the new independent variable the previous differential systems becomes

$$
\begin{gathered}
\frac{d L}{d g}=-\varepsilon \frac{C}{G} \frac{\partial\left(\mathcal{P}_{1}+\mathcal{P}_{2}\right)}{\partial l}+O\left(\varepsilon^{2}\right), \frac{d G}{d g}=-\varepsilon \frac{C}{G} \frac{\partial\left(\mathcal{P}_{1}+\mathcal{P}_{2}\right)}{\partial g}+O\left(\varepsilon^{2}\right), \\
\frac{d l}{d g}=\varepsilon \frac{C}{G} \frac{\partial\left(\mathcal{P}_{1}+\mathcal{P}_{2}\right)}{\partial L}+O\left(\varepsilon^{2}\right) .
\end{gathered}
$$

Since we want to study the periodic orbits on the energy level $\mathcal{H}=h$ we isolated $G$ from the energy, obtaining $G=\sqrt{2 h C}+O(\varepsilon)$. Therefore, the equations of motion on this energy level are

$$
\frac{d L}{d g}=-\varepsilon \sqrt{\frac{C}{2 h}} \frac{\partial\left(\mathcal{P}_{1}+\mathcal{P}_{2}\right)}{\partial l}+O\left(\varepsilon^{2}\right), \frac{d l}{d g}=\varepsilon \sqrt{\frac{C}{2 h}} \frac{\partial\left(\mathcal{P}_{1}+\mathcal{P}_{2}\right)}{\partial L}+O\left(\varepsilon^{2}\right) .
$$


These differential equations are in the normal form for applying the first order averaging theory, see Theorem 10. From this theorem the proof is completed.

Proof of Corollary 4. For proving this corollary using Theorem 3 we compute

$$
f_{1}=\left(f_{1}^{1}, f_{1}^{2}\right)=\left(\frac{\beta\left(L^{2}-2 C h\right) \sin 2 l}{\sqrt{2 C h}}, \frac{c H+2 h L(\beta \cos 2 l-1)}{2 \sqrt{2}(C h)^{3 / 2}}\right) .
$$

Note that the previous system can have the solutions

$$
\begin{gathered}
p_{1,2}=\left(L=-\sqrt{2 h C}, l= \pm \frac{1}{2} \operatorname{arcsec}\left(\frac{4 \beta h^{3 / 2}}{4 h^{3 / 2}+\sqrt{2 c} H}\right)\right), \\
p_{3,4}=\left(L=\sqrt{2 h C}, l= \pm \frac{1}{2} \operatorname{arcsec}\left(\frac{4 \beta h^{3 / 2}}{4 h^{3 / 2}-\sqrt{2 c} H}\right)\right) \\
p_{5,6}=\left(L=\frac{c H}{2 h(1+\beta)}, l= \pm \frac{\pi}{2}\right) \\
p_{7,8}=\left(L=\frac{c H}{2 h(1-\beta)}, l=0, \pi\right) .
\end{gathered}
$$

We note that the first four points are defined only if the arguments of the arcsec are in $(-\infty,-1) \cup(1,+\infty)$. The last four points are always defined because $\beta \in(0,1)$.

It is not difficult to compute the determinant (5) for this eight points and their eigenvalues, but since there are several possibilities we do not described all of them here.

Proof of Theorem 5. The Hamiltonian differential equations corresponding to the Hamiltonian (4) are

$$
\begin{array}{rlrl}
\frac{d L}{d t} & =-\varepsilon \frac{\partial \mathcal{P}_{1}}{\partial l}-\varepsilon^{2} \frac{\partial \mathcal{P}_{2}}{\partial l}, & & \frac{d G}{d t}=-\varepsilon \frac{\partial \mathcal{P}_{1}}{\partial g}-\varepsilon^{2} \frac{\partial \mathcal{P}_{2}}{\partial g} \\
\frac{d l}{d t}=\varepsilon \frac{\partial \mathcal{P}_{1}}{\partial L}+\varepsilon^{2} \frac{\partial \mathcal{P}_{2}}{\partial L}, & \frac{d g}{d t} & =\frac{G}{C}+\varepsilon \frac{\partial \mathcal{P}_{1}}{\partial G}+\varepsilon^{2} \frac{\partial \mathcal{P}_{2}}{\partial G} .
\end{array}
$$

Now taking $g$ as the new independent variable and isolating $G$ from the energy level $\mathcal{H}=h$ the previous differential system writes

$$
\begin{aligned}
& \frac{d L}{d g}=\varepsilon F_{11}+\varepsilon^{2} F_{12}+O\left(\varepsilon^{3}\right), \\
& \frac{d l}{d g}=\varepsilon F_{21}+\varepsilon^{2} F_{22}+O\left(\varepsilon^{3}\right),
\end{aligned}
$$

with

$$
F_{11}=-\frac{C}{a_{0}} \frac{\partial \mathcal{P}_{1}}{\partial l}, \quad F_{12}=\frac{C}{a_{0}} \frac{\partial \mathcal{P}_{1}}{\partial L}
$$




$$
\begin{gathered}
F_{21}=\frac{1}{a_{0}^{2}}\left(a_{1} C \frac{\partial \mathcal{P}_{1}}{\partial l}+C^{2} \frac{\partial \mathcal{P}_{1}}{\partial G} \frac{\partial \mathcal{P}_{1}}{\partial l}-a_{0} C \frac{\partial \mathcal{P}_{2}}{\partial l}-a_{0} a_{1} C \frac{\partial^{2} \mathcal{P}_{1}}{\partial l \partial G}\right), \\
F_{22}=\frac{1}{a_{0}^{2}}\left(-a_{1} C \frac{\partial \mathcal{P}_{1}}{\partial L}-C^{2} \frac{\partial \mathcal{P}_{1}}{\partial G} \frac{\partial \mathcal{P}_{1}}{\partial L}+a_{0} C \frac{\partial \mathcal{P}_{2}}{\partial L}+a_{0} a_{1} C \frac{\partial^{2} \mathcal{P}_{1}}{\partial L \partial G}\right),
\end{gathered}
$$

where in the previous functions we have

$$
G=a_{0}+a_{1} \varepsilon+O\left(\varepsilon^{2}\right), \quad a_{0}=\sqrt{2 C h}, \quad a_{1}=-\frac{C \mathcal{P}_{1}}{a_{0}} .
$$

Applying the averaging of first order described in Theorem 10 we obtain that the function $f_{1} \equiv 0$, due to the assumption (6). So we compute the function $f_{2}=\left(f_{2}^{1}, f_{2}^{2}\right)$ corresponding to the averaging of second order and we get the expression given in the statement of Theorem 5 .

Proof of Corollary 6. For proving this corollary using Theorem 5 we compute

$$
\begin{aligned}
f_{1} & =\left(f_{1}^{1}, f_{1}^{2}\right) \\
& =\left(\frac{\left(L^{2}-3 H^{2}\right) \beta \sin (2 l)}{\sqrt{3} H}, \frac{L\left(c^{2} C^{2}\left(9 H^{2}-7 L^{2}\right)+243 H^{6}(\beta \cos (2 l)-1)\right)}{243 \sqrt{3} H^{7}}\right) .
\end{aligned}
$$

Note that the previous system can have the solutions

$$
\begin{gathered}
p_{1,2,3,4}=\left(L= \pm \sqrt{3} H, l= \pm \frac{1}{2} \arccos \left(\frac{81 H^{4}+4 c^{2} C^{2}}{81 \beta H^{4}}\right)\right), \\
p_{5,6,7,8}=\left(L= \pm \frac{3 H \sqrt{c^{2} C^{2}-27 H^{4}(\beta+1)}}{\sqrt{7} c C}, l= \pm \frac{\pi}{2}\right), \\
p_{9,10,11,12}=\left(\begin{array}{c}
\left.L= \pm \frac{3 H \sqrt{c^{2} C^{2}+27 H^{4}(\beta-1)}}{\sqrt{7} c C}, l=0, \pi\right), \\
p_{13,14}=(L=0, l=0, \pi) .
\end{array},\right.
\end{gathered}
$$

We note that the first four points are defined only if the arguments of the arccos are in $[-1,1]$. The eight points from $p_{5}$ to $p_{12}$ are defined when the corresponding radical is non-negative. The last two points always are defined.

It is not difficult to compute the determinant (5) for this fourteen points and their eigenvalues, but since there are several possibilities we do not described all of them here.

\section{Proof of Theorem 7}

We summarize some facts on the Liouville-Arnold integrability theory for Hamiltonian systems and on the theory of averaging for periodic orbits of differential equations, for more details see $[1,2]$ and the subsection 7.1.2 of [2], respectively. Here we give the results only for Hamiltonian systems of two degrees of freedom, because the Hamiltonian systems of the rigid body with a fixed point under a Newtonian force field has two degrees of freedom, but we remark that these results work for an arbitrary number of degrees of freedom.

A Hamiltonian system with Hamiltonian $\mathcal{H}$ of two degrees of freedom is integrable in the sense of Liouville-Arnold if it has a second first integral $\mathcal{G}$ 
independent of $\mathcal{H}$ (i.e. the gradient vectors of $\mathcal{H}$ and $\mathcal{G}$ are independent in all the points of the phase space except perhaps in a set of zero Lebesgue measure), and in involution with $\mathcal{H}$ (i.e. the parenthesis of Poisson of $\mathcal{H}$ and $\mathcal{G}$ is zero).

A flow defined on a subspace of the phase space is complete if its solutions are defined for all time.

Now we are ready for stating the Liouville-Arnold's Theorem restricted to Hamiltonian systems of two degrees of freedom.

Theorem 8 (Lioville-Arnold). Suppose that a Hamiltonian system with two degrees of freedom defined on the phase space $M$ has its Hamiltonian $\mathcal{H}$ and the function $\mathcal{G}$ as two independent first integrals in involution. If $I_{h c}=\{p \in M$ : $H(p)=h$ and $C(p)=c\} \neq \emptyset$ and $(h, c)$ is a regular value of the map $(\mathcal{H}, \mathcal{G})$, then the following statements hold.

(a) $I_{h c}$ is a two dimensional submanifold of $M$ invariant under the flow of the Hamiltonian system.

(b) If the flow on a connected component $I_{h c}^{*}$ of $I_{h c}$ is complete, then $I_{h c}^{*}$ is diffeomorphic either to the torus $\mathbb{S}^{1} \times \mathbb{S}^{1}$, or to the cylinder $\mathbb{S}^{1} \times \mathbb{R}$, or to the plane $\mathbb{R}^{2}$. If $I_{h c}^{*}$ is compact, then the flow on it is always complete and $I_{h c}^{*} \approx \mathbb{S}^{1} \times \mathbb{S}^{1}$.

(c) Under the hypothesis (b) the flow on $I_{h c}^{*}$ is conjugated to a linear flow either on $\mathbb{S}^{1} \times \mathbb{S}^{1}$, on $\mathbb{S}^{1} \times \mathbb{R}$, or on $\mathbb{R}^{2}$.

We consider the autonomous differential system

$$
\dot{x}=f(x),
$$

where $f: U \rightarrow \mathbb{R}^{n}$ is $\mathcal{C}^{2}, U$ is an open subset of $\mathbb{R}^{n}$ and the dot denotes the derivative respect to the time $t$. We write its general solution as $\phi\left(t, x_{0}\right)$ with $\phi\left(0, x_{0}\right)=x_{0} \in U$ and $t$ belonging to its maximal interval of definition.

We say that $\phi\left(t, x_{0}\right)$ is $T$-periodic with $T>0$ if and only if $\phi\left(T, x_{0}\right)=x_{0}$ and $\phi\left(t, x_{0}\right) \neq x_{0}$ for $t \in(0, T)$. The periodic orbit associated to the periodic solution $\phi\left(t, x_{0}\right)$ is $\gamma=\left\{\phi\left(t, x_{0}\right), t \in[0, T]\right\}$. Note now that the variational equation associated to the $\mathrm{T}$-periodic solution $\phi\left(t, x_{0}\right)$ is

$$
\dot{M}=\left(\left.\frac{\partial f(x)}{\partial x}\right|_{x=\phi\left(t, x_{0}\right)}\right) M,
$$

where $M$ is an $n \times n$ matrix. The monodromy matrix associated to the $\mathrm{T}-$ periodic solution $\phi\left(t, x_{0}\right)$ is the solution $M\left(T, x_{0}\right)$ of $(7)$ satisfying that $M\left(0, x_{0}\right)$ is the identity matrix. The eigenvalues $\lambda$ of the monodromy matrix associated to the periodic solution $\phi\left(t, x_{0}\right)$ are called the multipliers of the periodic orbit.

For an autonomous differential system, one of the multipliers is always 1, and its corresponding eigenvector is tangent to the periodic orbit.

A periodic orbit of an autonomous Hamiltonian system always has two multipliers equal to one. One multiplier is 1 because the Hamiltonian system is 
autonomous, and the other has again value 1 due to the existence of the first integral given by the Hamiltonian.

Theorem 9 (Poincaré). If a Hamiltonian system with two degrees of freedom and Hamiltonian $H$ is Liouville-Arnold integrable, and $G$ is a second first integral such that the gradients of $H$ and $G$ are linearly independent at each point of a periodic orbit of the system, then all the multipliers of this periodic orbit are equal to 1.

Theorem 9 is due to Poincaré [11], see also [8]. It gives us a tool to study the non Liouville-Arnold integrability, independently of the class of differentiability of the second first integral. The main problem for applying this result in a negative way is to find periodic orbits having multipliers different from 1.

Proof of Theorem 7. By Theorem 1 (respectively 3 or 5) we assume that the Hamiltonian system at every positive Hamiltonian level of energy $h$ has periodic solutions corresponding to the zeros $\left(L_{0}, l_{0}\right)$ such that their associated Jacobians (5) are non-zero. Assuming that some of these Jacobians are different from 1 , the corresponding multipliers of the periodic orbits cannot be equal to one (see for more details the last part of the Appendix). Hence, by Theorem 9 either the the system cannot be Liouville-Arnold integrable with any second $\mathcal{C}^{1}$ first integral $G$, or this system is Liouville-Arnold integrable and the vector gradient of $H$ and $G$ are linearly dependent at some points of all these periodic orbits. This complete the proof.

\section{APPENDIX}

Now we shall present the basic results from averaging theory that we need for proving the results of this paper.

The next theorem provides a first and second order approximation for the periodic solutions of a periodic differential system, for the proof see Theorems 11.5 and 11.6 of Verhulst [12], [4] and [10] .

Consider the differential equation

$$
\dot{x}=\varepsilon F_{1}(t, x)+\varepsilon^{2} F_{2}(t, x)+\varepsilon^{3} R(t, x, \varepsilon), x(0)=x_{0}
$$

with $x \in D \subset \mathbb{R}^{n}, t \geq 0$. Moreover we assume that both $F_{1}(t, \mathrm{x})$ and $F_{2}(t, \mathrm{x})$ are $T$ periodic in $t$. Separately we consider in $D$ the averaged differential equation

$$
\dot{y}=\varepsilon f_{1}(y)+\varepsilon^{2} f_{2}(y), y(0)=x_{0},
$$

where

$$
\begin{aligned}
& f_{1}(y)=\frac{1}{T} \int_{0}^{T} F_{1}(t, y) d t . \\
& f_{2}(y)=\frac{1}{T} \int_{0}^{T}\left[D_{y} F_{1}(s, y) \int_{0}^{s} F_{1}(t, y) d t+F_{2}(s, t)\right] d s .
\end{aligned}
$$

Under certain conditions, equilibrium solutions of the averaged equation turn out to correspond with $T$-periodic solutions of equation (9). 
Theorem 10. Consider the two initial value problems (8) and (9). Suppose:

(i) $F_{1}$, its Jacobian $\partial F_{1} / \partial x$, its Hessian $\partial^{2} F_{1} / \partial x^{2}, F_{2}$ and its Jacobian $\partial F_{2} / \partial x$ are defined, continuous and bounded by an independent constant $\varepsilon$ in $[0, \infty) \times D$ and $\varepsilon \in\left(0, \varepsilon_{0}\right]$.

(ii) $F_{1}$ and $F_{2}$ are $T$-periodic in $t$ ( $T$ independent of $\varepsilon$ ).

(iii) $y(t)$ belongs to $\Omega$ on the interval of time $[0,1 / \varepsilon]$.

Then the following statements hold.

(a) For $t \in[1, \varepsilon]$ we have that $x(t)-y(t)=O(\varepsilon)$, as $\varepsilon \rightarrow 0$.

(b) If $p$ is a singular point of the averaged equation (9) and

$$
\left.\operatorname{det}\left(\frac{\partial\left(f_{1}+\varepsilon f_{2}\right)}{\partial y}\right)\right|_{\mathrm{y}=p} \neq 0,
$$

then there exists a T-periodic solution $\varphi(t, \varepsilon)$ of equation (8) which is close to $p$ such that $\varphi(0, \varepsilon) \rightarrow p$ as $\varepsilon \rightarrow 0$.

(c) The stability or instability of the limit cycle $\varphi(t, \varepsilon)$ is given by the stability or instability of the singular point $p$ of the averaged system (9). In fact, the singular point $p$ has the stability behavior of the Poincaré map associated to the limit cycle $\varphi(t, \varepsilon)$.

Remark 11. If the function $f_{1}$ is not identically zero, then the zeros of $f_{1}+\varepsilon f_{2}$ are mainly the zeros of $f_{1}$ for $\varepsilon$ sufficiently small. In this case the previous theorem provides the so-called averaging theory of first order.

If the function $f_{1}$ is identically zero and $f_{2}$ is not identically zero, then the zeros of $f_{1}+\varepsilon f_{2}$ are the zeros of $f_{2}$. In this case the previous theorem provides the so-called averaging theory of second order.

We point out the main facts in order to prove Theorem 10(c), for more details see section 6.3 and 11.8 in [12]. Suppose that $\varphi(t, \varepsilon)$ is a periodic solution of (8) corresponding to $y=p$ an equilibrium point of the averaged system (9). Linearizing equation (8) in a neighborhood of the periodic solution $\varphi(t, \varepsilon)$ we obtain a linear equation with $T$-periodic coefficients

$$
\dot{x}=\varepsilon A(t, \varepsilon) x, \quad A(t, \varepsilon)=\frac{\partial}{\partial x}\left[\left.F\left(_{1}(t, x)-F_{2}(t, x, \varepsilon)\right]\right|_{x=\varphi(t, \varepsilon)} .\right.
$$

We introduce the $T$-periodic matrices

$$
B(t)=\frac{\partial F_{1}}{\partial x}(t, p), \quad B_{1}=\frac{1}{T} \int_{0}^{T} B(t) d t, \quad C(t)=\int_{0}^{t}\left(B(s)-B_{1}\right) d s .
$$

From Theorem 10 we have

$$
\lim _{\varepsilon \rightarrow 0} A(t, \varepsilon)=B(t)
$$

and it is clear that $B_{1}$ is the matrix of the linearized averaged equation. The matrix $C$ has average zero. The near identity transformation

$$
x \longmapsto y=(I-\varepsilon C(t)) x,
$$

permits to write $(10)$ as

$$
\dot{y}=\varepsilon B_{1} y+\varepsilon[A(t, \varepsilon)-B(t)] y+O\left(\varepsilon^{2}\right) .
$$


Notice that $A(t, \varepsilon)-B(t) \rightarrow 0$ as $\varepsilon \rightarrow 0$, and also the characteristic exponents of equation (12) depend continuously on the small parameter $\varepsilon$. It follows that, for $\varepsilon$ sufficiently small, if the determinant of $B_{1}$ is not zero, then 0 is not an eigenvalue of the matrix $B_{1}$ and then it is not a characteristic exponent of (12). By the near-identity transformation we obtain that system (10) has not multipliers equal to 1.

\section{ACKNOWLEDGEMENTS}

The first author was partially supported by MCYT/FEDER grant number MTM2011-22587, Junta de Comunidades de Castilla-La Mancha, grant number PEII09-0220-0222. The second author was partially supported by MICINN/FEDER grant number MTM2008-03437, by AGAUR grant number 2009SGR 410 and ICREA Academia. The third author was partially supported by Fundación Séneca de la Región de Murcia grant number 12001/PI/09.

\section{REFERENCES}

[1] R. Abraham and J.E. Marsden, Foundations of Mechanics, Benjamin, Reading, Masachusets, 1978.

[2] V.I. Arnold, V. Kozlov and A. Neishtadt, Dynamical Systems III. Mathematical Aspects of Classical and Celestial Mechanics, Third Edition, Encyclopaedia of Mathematical Science, Springer, Berlin, 2006.

[3] A.V. Borisov And I.S. Mamaev, Dynamics of the rigid body, Izhevsk, RCD, 2001.

[4] A. Buica And J. Llibre, Averaging methods for finding periodic orbits via Brouwer degree, Bulletin de Kinoshitas Sciences Mathemàtiques 128 (2004), 7-22.

[5] L. Jiménez-Lara and J. Llibre, Periodic orbits and non-Integrability of HenonHeiles system, J. Physics A: Maths. Gen., 44 (2011), 205103-14 pp.

[6] L. Jiménez-Lara And J. Llibre, Periodic orbits and non-integrability of generalized classical Yang-Mills Hamiltonian system, J. Math. Phys., 52 (2011), 032901-9 pp.

[7] H. Kinoshita, First order perturbations of the two finite body problem, Publications of the Astronomical Society of Japan, 24, (1972), 423-457.

[8] V.V. KozLov, Integrability and non-integrability in Hamiltonian mechanics, Russian Math. Surveys, 38(1), (1983), 1-76.

[9] E. Leimanis, The general problem of the motion of coupled rigid bodies about a fixed point, Springer Verlag Berlin, 1965.

[10] J. LliBRe, Averaging theory and limit cycles for quadratic systems, Radovi Matematicki 11, (2002), 215-228.

[11] H. Poincaré, Les méthodes nouvelles de la mécanique céleste, Vol. I, Gauthier-Villars, Paris 1899.

[12] F. Verhulst, Nonlinear Differential Equations and Dynamical Systems, Universitext, Springer, 1991.

${ }^{1}$ Departamento de Matemática Aplicada y Estadística. Universidad Politécnica de Cartagena, Hospital de Marina, 30203-Cartagena, Región de Murcia, SPAIN

E-mail address: juan.garcia@upct.es

2 Departament de Matemàtiques. Universitat Autònoma de Barcelona, Bellaterra, 08193-Barcelona, Catalonia, Spain

E-mail address: jllibre@mat.uab.cat 
3 Centro Universitario de la Defensa. Academia General del Aire. Universidad Politécnica de Cartagena, 30720-Santiago de la Ribera, Región de Murcia, Spain

E-mail address: juanantonio.vera@cud.upct.es 